Supporting information

\title{
Simple Is Best: A $p$-Phenylene Bridging Methoxydiphenylamine-Substituted Carbazole Hole Transporter for High-Performance Perovskite Solar Cells
}

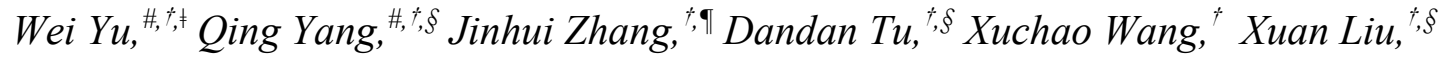

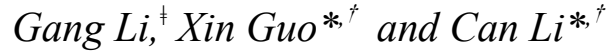

`State Key Laboratory of Catalysis, Dalian National Laboratory for Clean Energy, Dalian Institute of Chemical Physics, Chinese Academy of Sciences, Dalian 116023, China

${ }^{\ddagger}$ Department of Electronic and Information Engineering, The Hong Kong Polytechnic University, Hung Hom, Kowloon, Hong Kong SAR, China

$\S$ University of Chinese Academy of Sciences, Beijing 100049, China

" Department of Materials Science and Engineering, Dalian Maritime University,

Dalian, 116023, China

\# These authors contributed equally to this work.

\section{Corresponding Author}

*Email: guoxin@dicp.ac.cn; canli@dicp.ac.cn. 


\section{Contents}

Hole Mobility and conductivity Measurements …………………….......... S2

Synthetic routes and cost estimation ...................................................... S3

Density functional theory calculation of $p \mathrm{Ph}-2 \mathrm{MODPACz} \ldots \ldots \ldots \ldots \ldots \ldots \ldots \ldots . . . . . . . . . . . . . . .54$

TGA and DSC curves of $p P h-2 M O D P A C z$............................................. 4

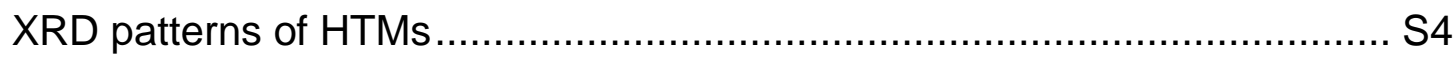

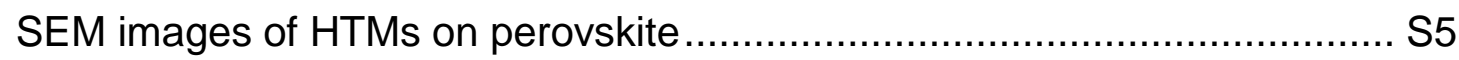

Statistical device performance distribution for pPh-2MODPACz .................. S5

Hysteresis behavior test for pPh-2MODPACz ………............................ S5

Steady-state PL spectra of perovskite films with and without HTMs atop.... S6

Time-resolved PL decay spectra of perovskite films with and without HTMs S6 stability tests of pPh-2MODPACz and spiro-OMeTAD based devices........S7

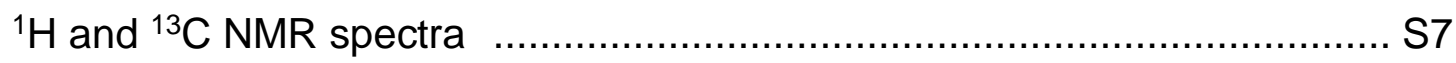




\section{Hole Mobility Measurements}

Hole mobility was investigated by the method of space-charge limited current (SCLC). The hole-only devices were fabricated with a structure of ITO/PEDOT:PSS/HTM/MoO $/ \mathrm{Au}$, then the $J-V$ results were fitted using the Mott-Gurney law that includes field-dependent mobility, ${ }^{1}$ given by the following Equation (1),

$$
J=\frac{9}{8} \varepsilon_{0} \varepsilon_{r} \mu_{0} \frac{\left(V-V_{b i}\right)^{2}}{d^{3}} \exp \left(\beta \sqrt{\frac{V-V_{b i}}{d}}\right)
$$

where $\varepsilon_{0} \varepsilon_{r}$ is the dielectric permittivity of the active layer, $d$ is the thickness of the active layer, $\mu_{0}$ the zero-field mobility, and $\beta$ the field activation factor.

\section{Conductivity Measurements}

The conductivities of the HTMs were determined by using a two-contact electrical conductivity set-up, which were performed by the following procedures. ${ }^{2}$ Glass substrates were carefully cleaned in ultrasonic baths of isopropanol and ethanol for 30 min successively and then dried by flowing nitrogen gas. A thin layer of compact $\mathrm{TiO}_{2}$ was coated on the glass substrates by spin-coating the titanium isopropoxide solution. The thickness of the film is $c a$. $40 \mathrm{~nm}$. After sintering the $\mathrm{TiO}_{2}$ film on a hotplate at $500{ }^{\circ} \mathrm{C}$ for $30 \mathrm{~min}$, the film was cooled down to room temperature, then a solution of HTM in chlorobenzene was spin-coated. The concentrations and additives were the same as those used in devices. Subsequently, a $200 \mathrm{~nm}$ of Ag back contact was deposited onto the HTM 
layer by thermal evaporation in vacuum chamber with a base pressure of $<$ $6 \times 10^{-4} \mathrm{~Pa} . J-V$ characteristics were recorded on a Keithley 4200 Semiconductor Characterization System.

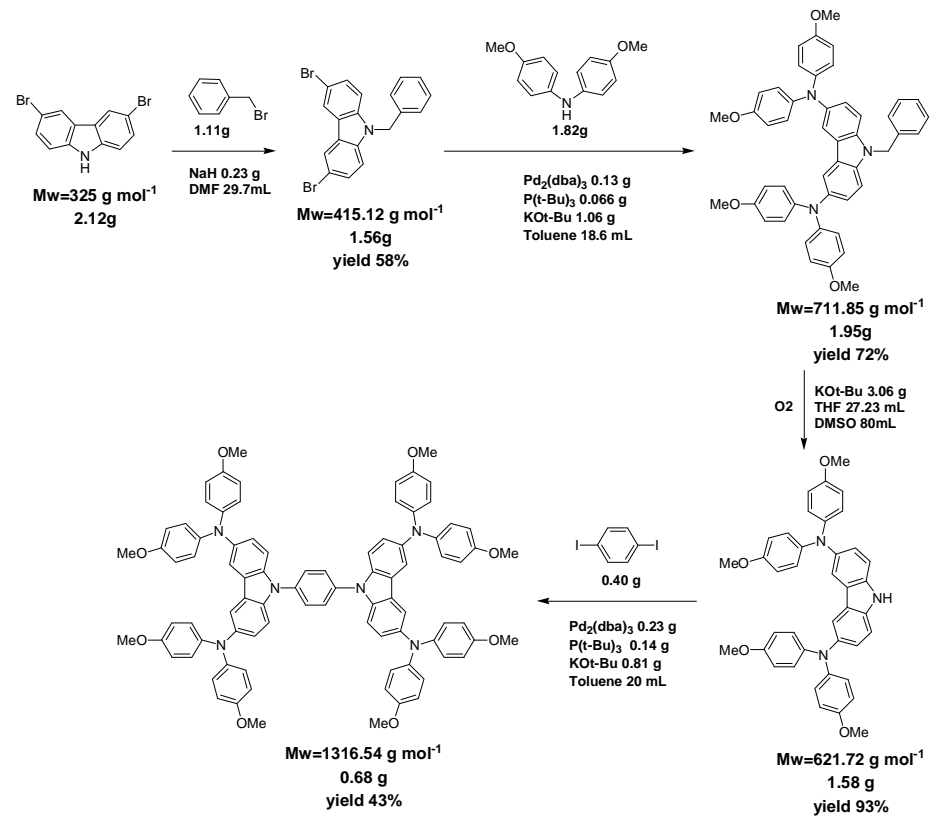

Scheme S1. Detailed synthetic procedures of $p \mathrm{Ph}-2 \mathrm{MODPACz}$ for cost estimations.

Table S1. Materials quantities and cost for the synthesis of $p \mathrm{Ph}-2 \mathrm{MODPACz}$.

\begin{tabular}{|c|c|c|c|c|}
\hline Chemical name & Weight of reagent $(\mathrm{g})$ & Price $(\$ / g)$ & Cost $(\$)$ & Cost $(\$ / g)$ \\
\hline 3,6-dibromocarbazole & 2.12 & 0.830 & 1.760 & \\
\hline $\mathrm{NaH}$ & 0.23 & 0.455 & 0.105 & \\
\hline DMF & 28.04 & 0.0055 & 0.154 & \\
\hline Benzyl bromide & 1.11 & 0.610 & 0.680 & \\
\hline 4,4'-dimethoxydiphenylamine & 1.82 & 4.640 & 8.440 & \\
\hline $\mathrm{t}-\mathrm{BuOK}$ & 4.93 & 0.150 & 0.740 & \\
\hline $\mathrm{Pd}_{2}(\mathrm{dba})_{3}$ & 0.36 & 14.150 & 5.094 & \\
\hline $\mathrm{P}(\mathrm{t}-\mathrm{Bu})_{3} \mathrm{HBF}_{4}$ & 0.206 & 5.800 & 1.195 & \\
\hline Toluene & 33.47 & 0.0065 & 0.217 & \\
\hline DMSO & 88.00 & 0.0100 & 0.880 & \\
\hline Tetrahydrofuran & 24.03 & 0.0120 & 0.288 & \\
\hline 1,4-diiodobenzene & 0.40 & 1.800 & 0.720 & \\
\hline $\mathrm{CH}_{2} \mathrm{Cl}_{2}$ & 100 & 0.0043 & 0.430 & \\
\hline Hexane & 100 & 0.011 & 1.100 & \\
\hline Ethyl acetate & 100 & 0.0048 & 0.480 & \\
\hline $\mathrm{MgSO}_{4}$ & 20 & 0.0049 & 0.098 & \\
\hline pPh-2MODPACz & 0.68 & & 22.381 & 32.91 \\
\hline
\end{tabular}



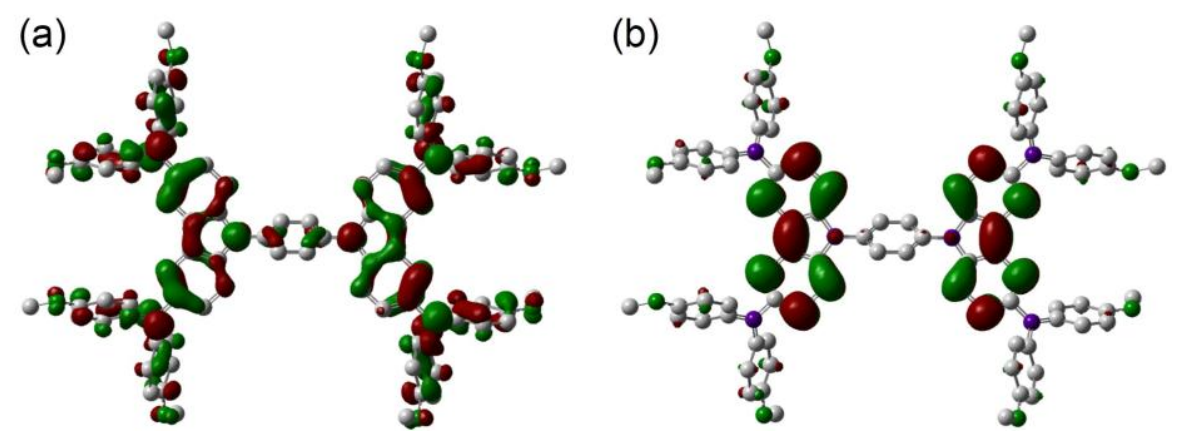

Figure S1. Electronic density distributions of HOMO (a) and LUMO (b) for $p \mathrm{Ph}$ 2MODPACz, calculated with Gaussian at the B3LYP/6-31G(d) level of theory.
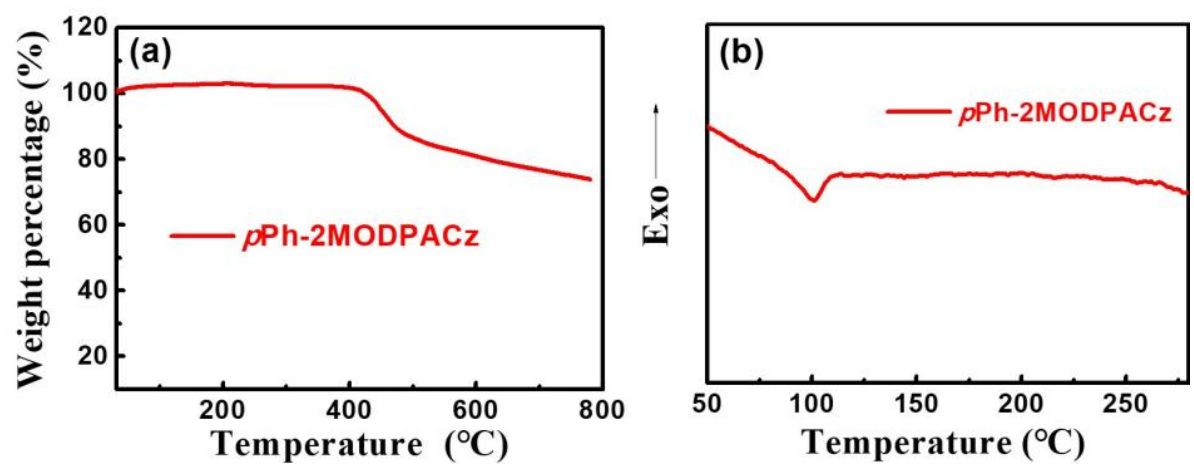

Figure S2. (a) TGA and (b) DSC curves of $p$ Ph-2MODPACz.

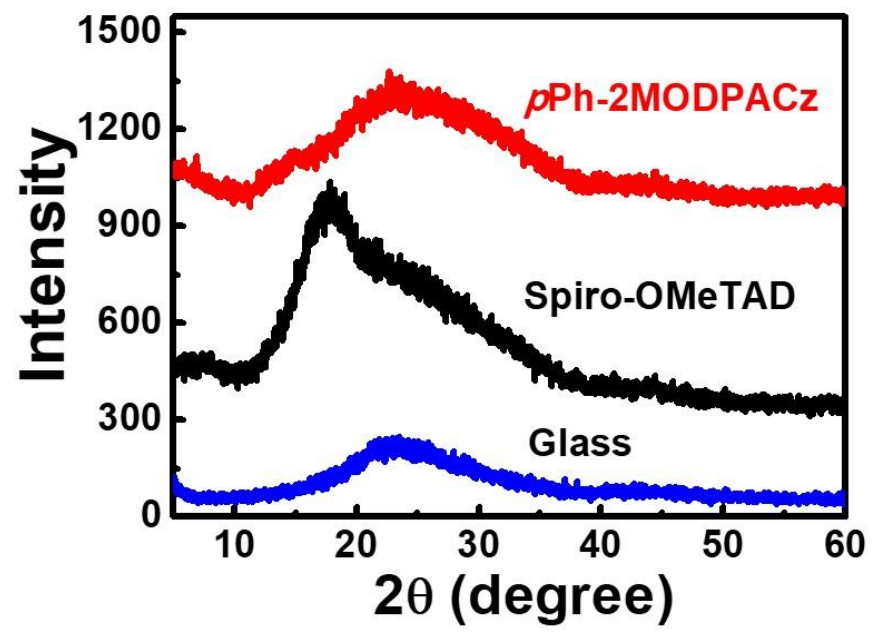

Figure S3. XRD patterns of $p \mathrm{Ph}-2 \mathrm{MODPACz}$ and spiro-OMeTAD films on glass substrates. 

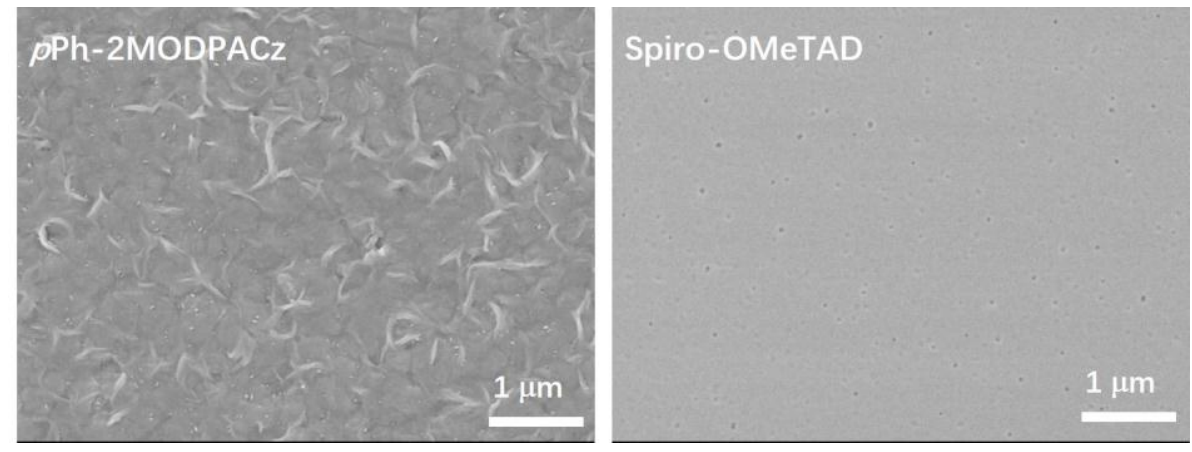

Figure S4. SEM images of $p \mathrm{Ph}-2 \mathrm{MODPACz}$ and spiro-OMeTAD on perovskite.
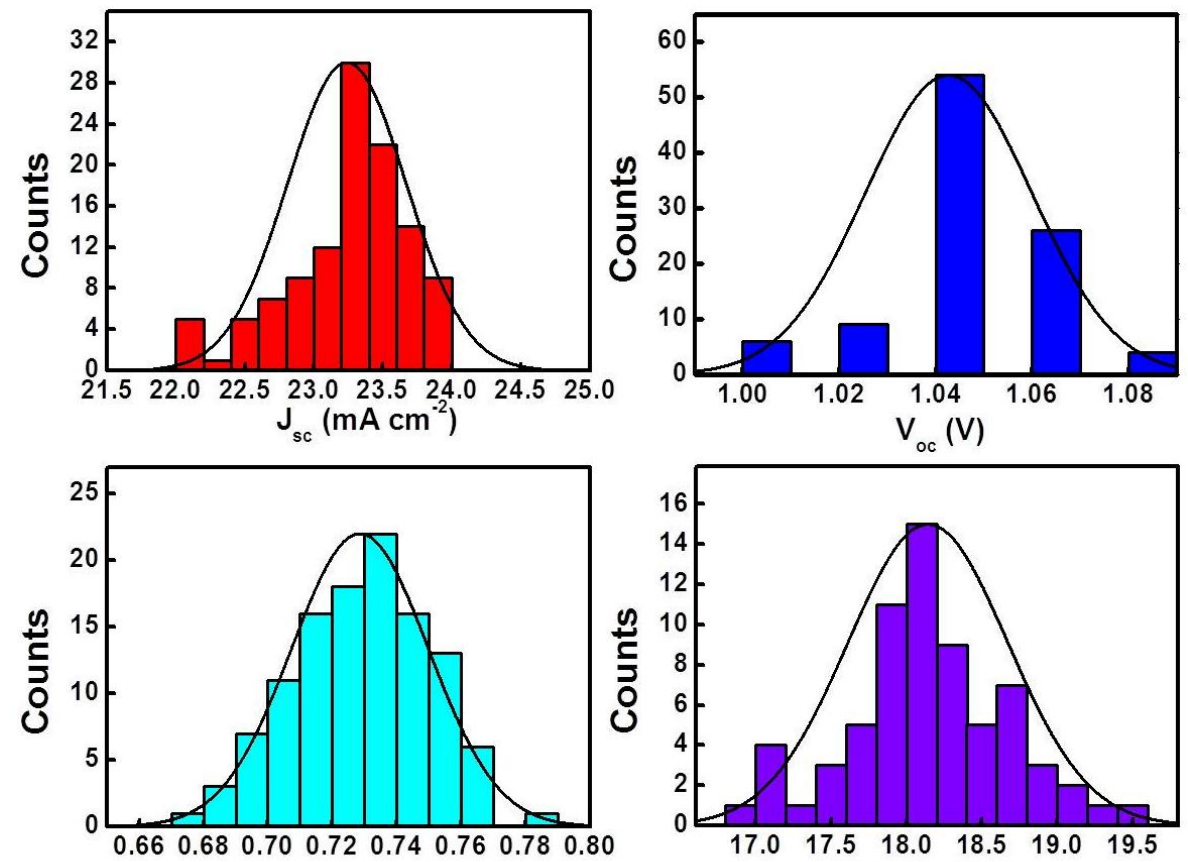

FF

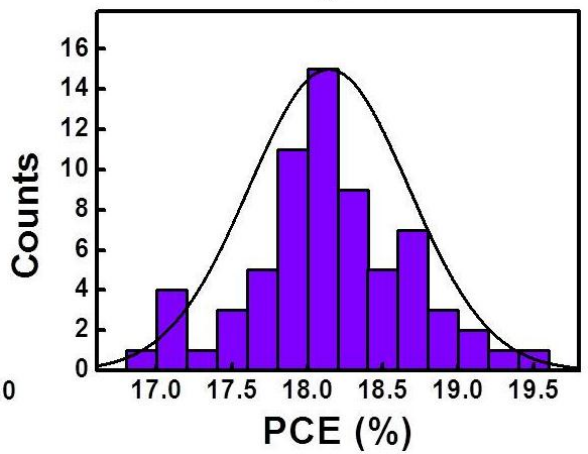

Figure S5. Statistical device parameters for $p \mathrm{Ph}-2 \mathrm{MODPACz}$-based PSCs measured

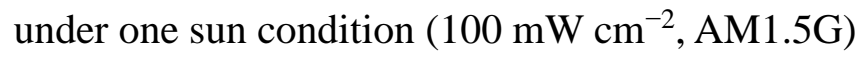
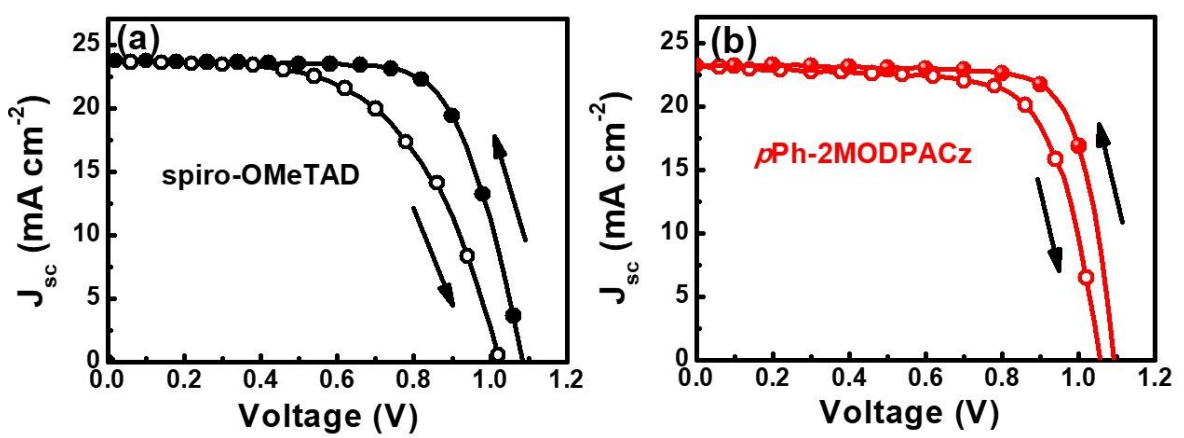

Figure S6. $J-V$ characteristics of PSCs using (a) Spiro-OMeTAD and (b) $p \mathrm{Ph}$ 2MODPACz as HTMs under different scan conditions. 
Table S2. Device parameters of PSCs with spiro-OMeTAD and $p \mathrm{Ph}-2 \mathrm{MODPACz}$ as HTMs, measured by reverse and forward scans.

\begin{tabular}{ccccccc}
\hline HTMs & HIs & Scan direction & $J_{s c}\left(\mathrm{~mA} \mathrm{~cm}^{-2}\right)$ & $V_{o c}(\mathrm{~V})$ & FF & PCE $(\%)$ \\
\hline \multirow{2}{*}{ Spiro-OMeTAD } & \multirow{2}{*}{0.26} & Reverse & 23.05 & 1.08 & 0.72 & 17.92 \\
& & Forward & 22.99 & 1.02 & 0.58 & 13.60 \\
\hline$p$ Ph- & \multirow{2}{*}{0.07} & Reverse & 23.22 & 1.09 & 0.78 & 19.74 \\
2 MODPACz & & Forward & 23.12 & 1.05 & 0.72 & 17.48 \\
\hline
\end{tabular}

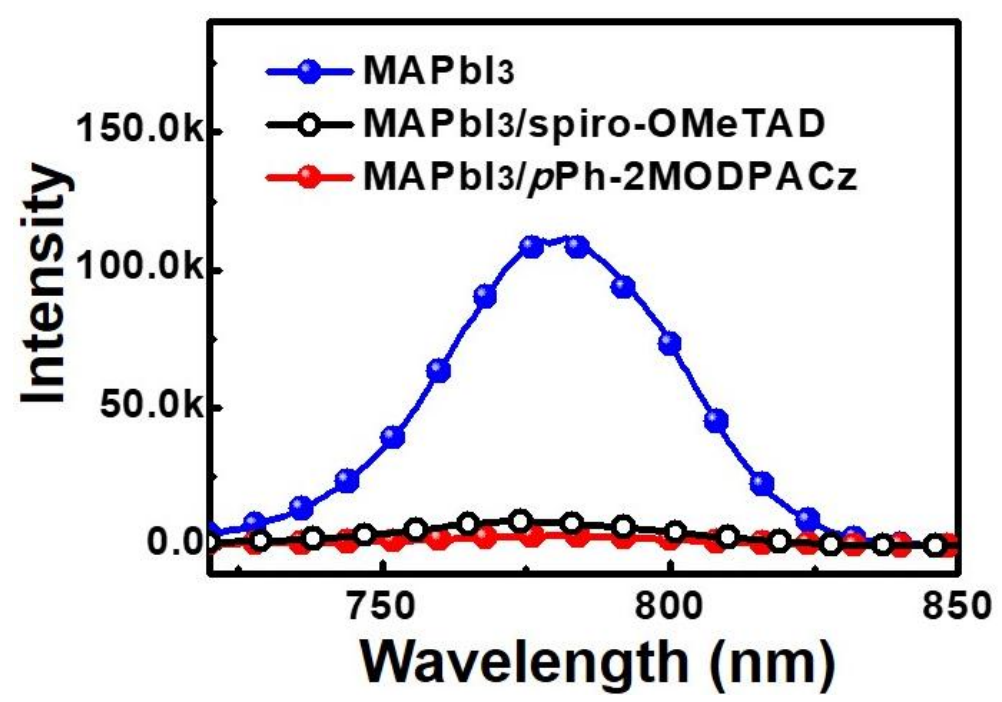

Figure S7. PL decay spectra of $\mathrm{MAPbI}_{3}$ films with and without HTMs atop.

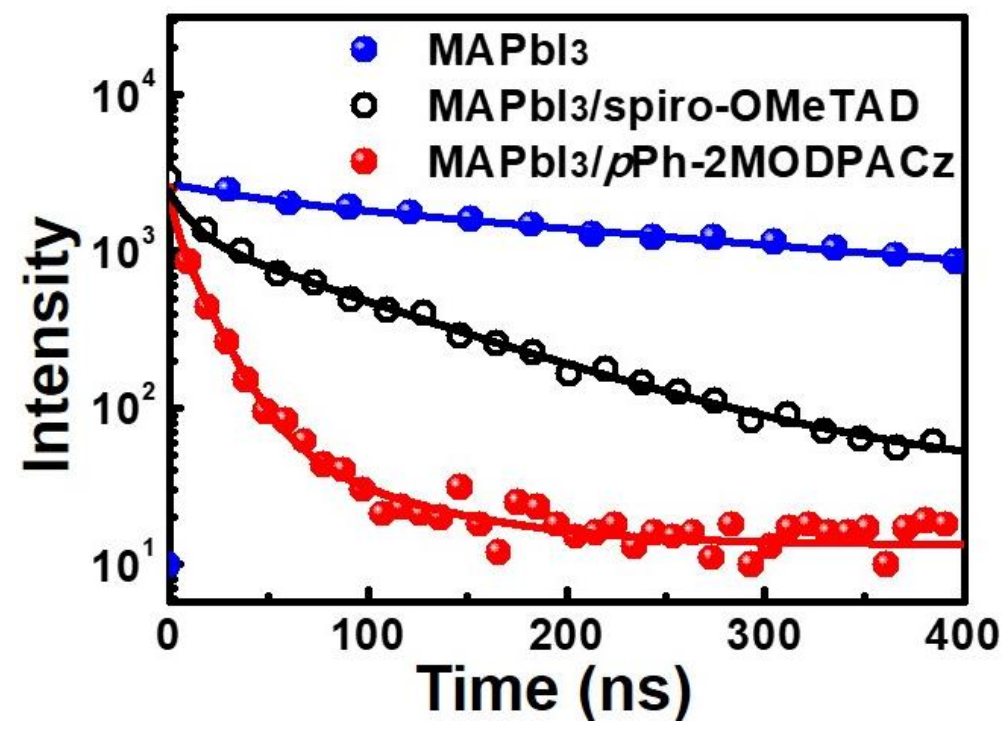

Figure S8. Time-resolved PL decay spectra of $\mathrm{MAPbI}_{3}$ films with and without undoped HTMs atop. 
Table S3. Fitted PL decay lifetimes of $\mathrm{MAPbI}_{3}$ films with and without HTMs.

\begin{tabular}{cccccc}
\hline Samples & $\tau_{1}(\mathrm{~ns})$ & $\mathrm{A}_{1}(\%)$ & $\tau_{2}(\mathrm{~ns})$ & $\mathrm{A}_{2}(\%)$ & $\tau_{\mathrm{ave}}(\mathrm{ns})$ \\
\hline Glass/MAPbI $_{3}$ & 96.23 & 5.07 & 450.42 & 94.93 & 432.46 \\
Glass/MAPbI $/$ /spiro-OMeTAD & 14.87 & 41.19 & 50.30 & 58.81 & 35.74 \\
Glass/MAPbI $/ 2$ PPh-2MODPACz & 9.74 & 44.61 & 37.34 & 55.39 & 25.03 \\
\hline
\end{tabular}

The TRPL spectra were fitted by a bi-exponential Equation (2),

$f(t)=B+A_{1} \exp \left(-\frac{t}{\tau_{1}}\right)+A_{2} \exp \left(-\frac{t}{\tau_{2}}\right)$

where $A_{1}, A_{2}$ are time independent coefficients of amplitude fraction for each decay component and $\tau_{1}, \tau_{2}$ represent the decay time.
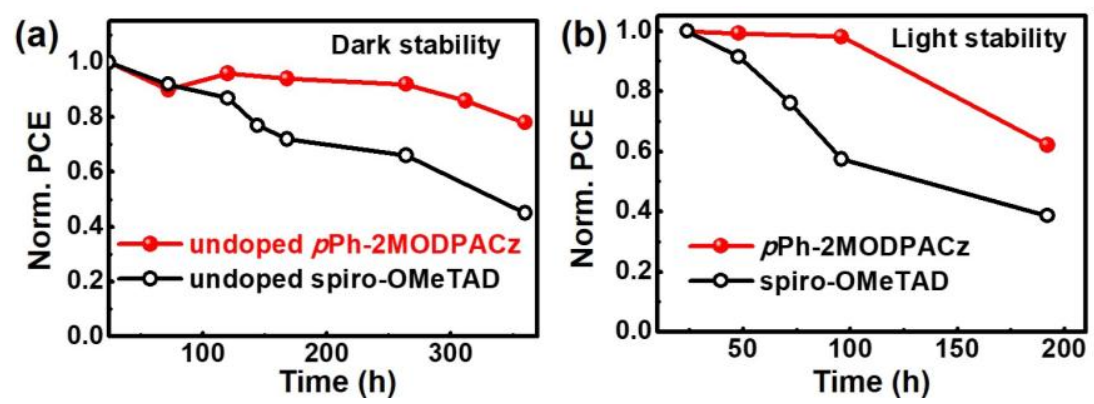

Figure S9. Stability tests of (a) undoped HTM-based devices without encapsulation keep in glove box and (b) $p$ Ph-2MODPACz and spiro-OMeTAD based devices without encapsulation under continuous one sun light irradiation.

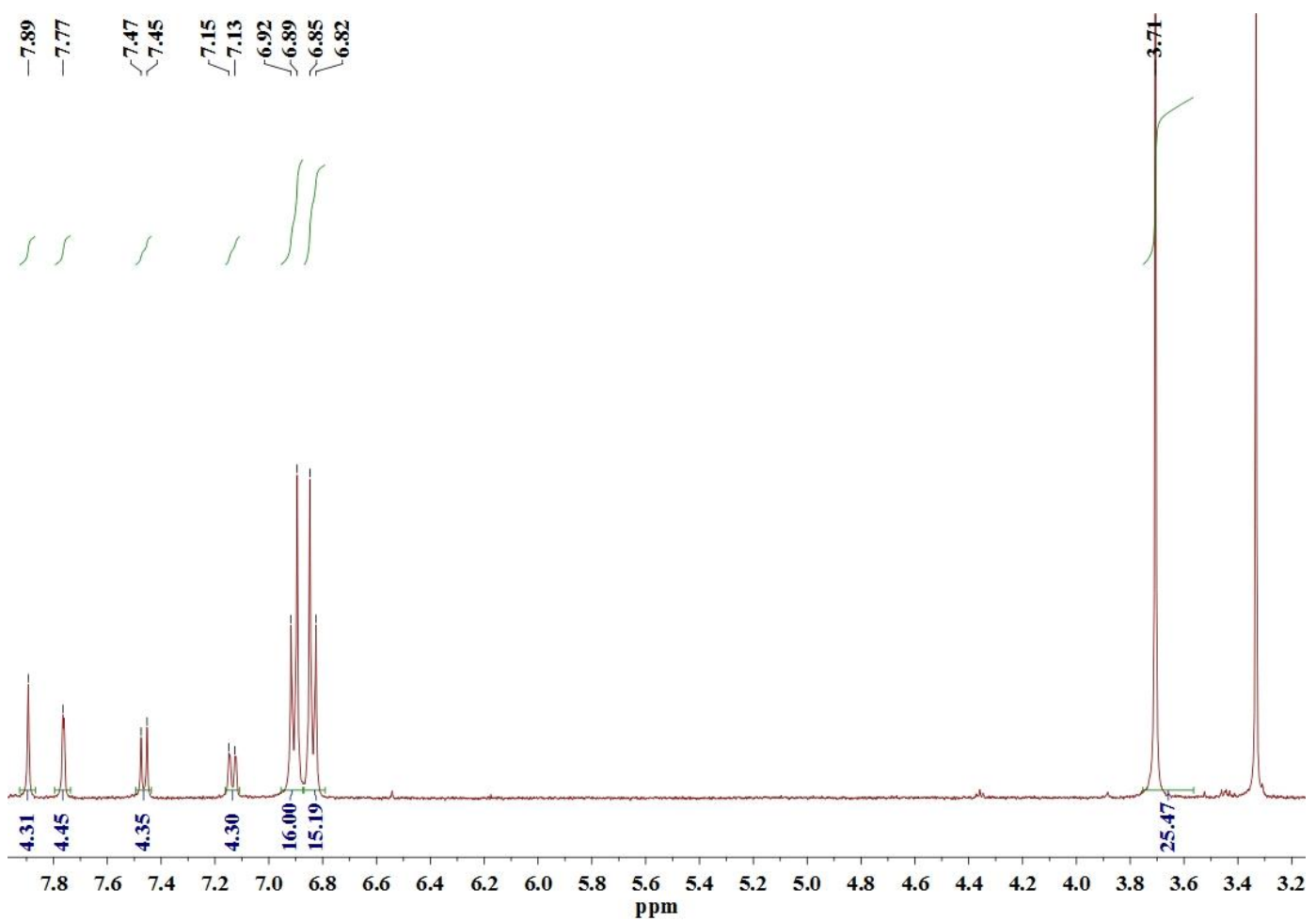

Figure S10. ${ }^{1} \mathrm{H}$ NMR spectrum of $p \mathrm{Ph}-2 \mathrm{MODPACz}$. 


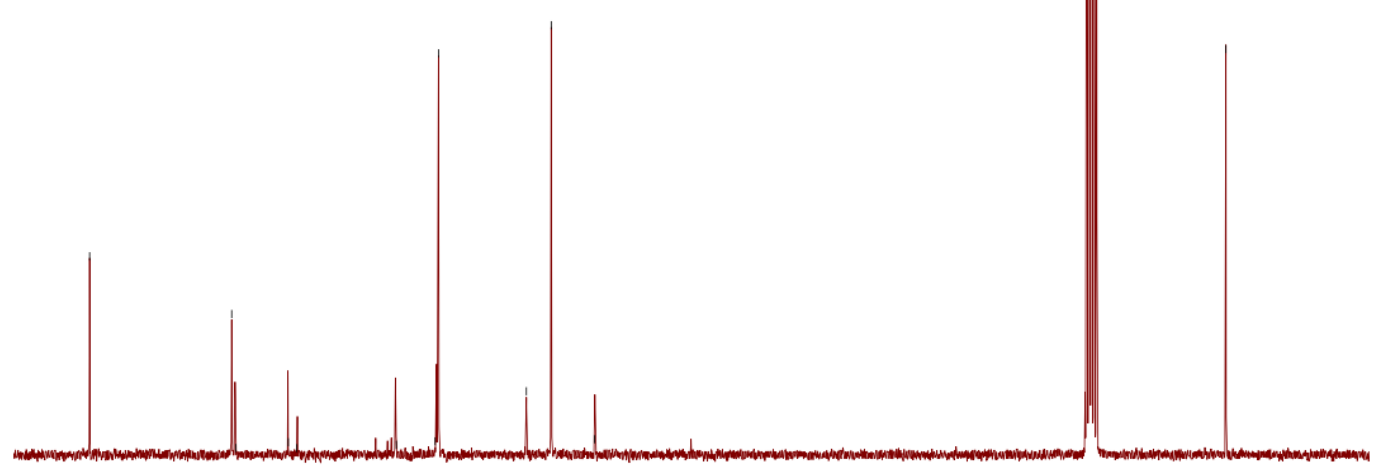

$\begin{array}{lllllllllllllllllllllll}155 & 150 & 145 & 140 & 135 & 130 & 125 & 120 & 115 & 110 & 105 & 100 & 95 & 90 & 85 & 80 & 75 & 70 & 65 & 60 & 55 & 50 & 45\end{array}$

Figure S11. ${ }^{13} \mathrm{C}$ NMR spectrum of $p$ Ph-2MODPACz.

\section{References}

(1) Murgatroyd, P. N., Theory of space-charge-limited current enhanced by Frenkel effect. J. Phys. D: Appl. Phys. 1970, 3, 151-156.

(2) Zhang, J.; Xu, B.; Johansson, M. B.; Vlachopoulos, N.; Boschloo, G.; Sun, L.; Johansson, E. M. J.; Hagfeldt, A., Strategy to Boost the Efficiency of Mixed-Ion Perovskite Solar Cells: Changing Geometry of the Hole Transporting Material. ACS Nano 2016, 10 (7), 6816-6825. 